\title{
Photonic Delay Line for Broadband Optical Signals, based on Dynamic Grating Reflectors in Fibers
}

\author{
Sanghoon Chin, Nikolay Primerov and Luc Thevenaz \\ Ecole Polytechnique Fédérale de Lausanne, Institute of Electrical Engineering, \\ STI GR-SCI-LT Station 11, CH-1015 Lausanne, Switzerland, sanghoon.chin@epfl.ch
}

\begin{abstract}
We experimentally demonstrate that a novel type of photonic delay line based on movable dynamic grating reflectors generated in polarization maintaining fibers can provide continuously tunable signal delaying for high capacity optical data streams and wide bandwidth microwave signals.
\end{abstract}

\section{Introduction}

Dynamic control of optical signal delays has rapidly developed and been experimentally demonstrated over the last decade since it has been proved to be a promising solution for alloptical digital and analogue signal processing in modern optical communication systems. Most existing photonic delay lines, however, relied on two main techniques ${ }^{1}$. First, slow and fast lightbased delaying scheme offers a flexible timing tool, but the maximum achievable time delay is inherently limited due to a large dispersion induced by a spectral resonance, presenting a critical bottleneck in terms of optical storage capacity $^{2-3}$. Secondly, dispersive delay line based on group velocity dispersion in optical media was proposed and improved by a large increment the fractional delay ${ }^{4-6}$. However, this scheme still suffers from signal distortion since the chromatic dispersion is a key parameter to control the amount of time delay, so limiting the signal bandwidth. As a matter of fact, producing a large amount of signal delay with negligible distortion remains still a scientific challenge.

We recently proposed a new architecture to achieve signal delaying and demonstrated that movable dynamic Brillouin gratings implemented in polarization maintaining fibers (PMF) can reflect an isolated signal pulse at any preset position along the fiber, resulting in a large signal delay with moderate signal distortion ${ }^{7}$. In this paper, we present further study and development on this technique, particularly its adequacy for delaying real data streams and broadband microwave signals.

\section{Principle}

The technique relies on the fact that a positionadjustable dynamic acousto-optic grating along a high birefringence fiber (PMF) can be generated by ordinary stimulated Brillouin scattering (SBS) through the interaction of two pump pulses counter-propagating along the fiber, as already described in our previous paper ${ }^{7}$. The acoustic wave builds up at the pulse crossing point as long as the pulses physically overlap and then gradually decays through acoustic dissipative losses. It means that the fast decaying feature of acoustic phonons makes such a grating non permanent and acts as a serious penalty for such a delay line, in terms of the total length of the data packet carrying the information. Actually, the packet length cannot exceed the acoustic lifetime $\tau_{B}$, showing a typical value of $10 \mathrm{~ns}$ in standard single mode fibers. This time constant $\tau_{B}$ varies with intrinsic parameters of materials such as the viscosity coefficient in the medium and the incident frequency of pump wave, but remains of the same order of magnitude for all glass materials with potentialities in modern photonics. On the other hand, the dynamic grating can be periodically regenerated at a desired position, by simply using a sequence of pump pulses at a repetition rate faster than the acoustic lifetime, so that it can be rendered quasi-permanent and kept localized in the fiber.

Fig. 1 depicts the principle of the generation of quasi-permanent dynamic gratings, in the configuration we implemented for the demonstration. Two counter-propagating pulse trains with 600 ps FWHM duration at distinct frequencies $v_{1}$ and $v_{2}\left(v_{2}>v_{1}\right)$ are used as Brillouin pumps. The two pulses are spectrally separated by the Brillouin frequency shift $v_{B}$, so that a dynamic grating is generated at the pulses crossing point within the fiber through SBS interaction. The repetition rate of the pulse trains is properly adjusted at $8 \mathrm{~ns}$ shorter than

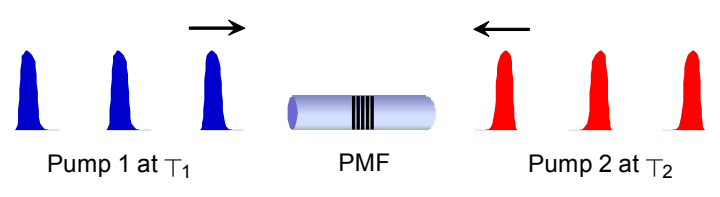

Fig. 1: Configuration of pump pulses to generate a quasi-permanent dynamic grating in PM fibers. 


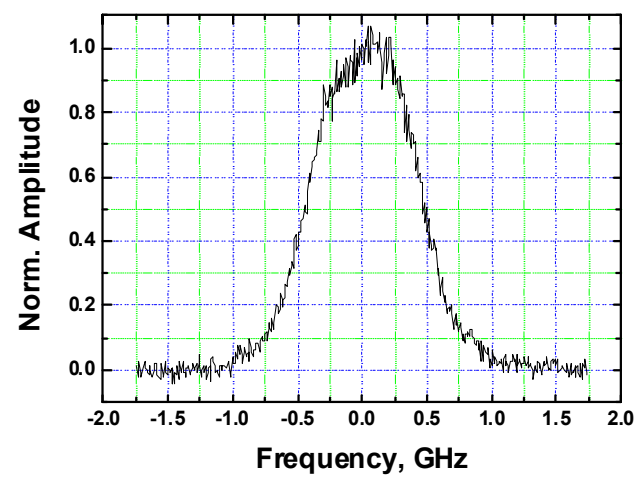

Fig. 2: Reflected power of a frequency-swept probe, representing the reflection spectrum of the dynamic Brillouin grating.

the $10 \mathrm{~ns}$ acoustic lifetime and longer than the $3 \mathrm{~ns}$ transit time through the $60 \mathrm{~cm}$-long PMF. This way, it is secured that only one dynamic grating is present along the entire $60 \mathrm{~cm}$ PM fiber and is periodically refreshed before it vanishes.

We first measured the spectral reflection of the generated dynamic grating. The states of polarization of the two counter-propagating pump pulses are linearly polarized along the slow axis of the PMF before entering the fiber. A continuous probe wave linearly polarized along the fast axis is simultaneously launched into the fiber via a circulator and its frequency is swept through the reflection band of the dynamic grating. The central frequency of the dynamic grating was measured to be $43 \mathrm{GHz}$ above the Pump 2 frequency $v_{2}$. A fraction of the probe light reflected by the dynamic grating is then detected and displayed on an oscilloscope as a function of frequency, as shown in Fig. 2. The bandwidth of the grating was measured to be $\sim 1 \mathrm{GHz}$ at full width at half maximum. However, it must be pointed out that the reflection bandwidth in this delay line is by essence given by the spatial length of the grating, actually corresponding to the half length of the pump pulses duration.

\section{Experimental setup and Results}

Fig. 3 depicts a simplified schematic diagram of the experimental implementation to realize signal delaying. A single distributed feedback (DFB) laser diode operating at $1551 \mathrm{~nm}$ is used to generate the two pump pulse trains, the distinct frequencies being synthesized using the traditional modulation scheme $^{8}$. This way a highly stable frequency difference between the two pumps is obtained. As Brillouin gain medium a $2 \mathrm{~m}$-long PANDA type PM fiber is used, showing a Brillouin frequency shift of $10.87 \mathrm{GHz}$ and a Brillouin gain bandwidth of $30 \mathrm{MHz}$. Actually, according to the $8 \mathrm{~ns}$ repetition rate of

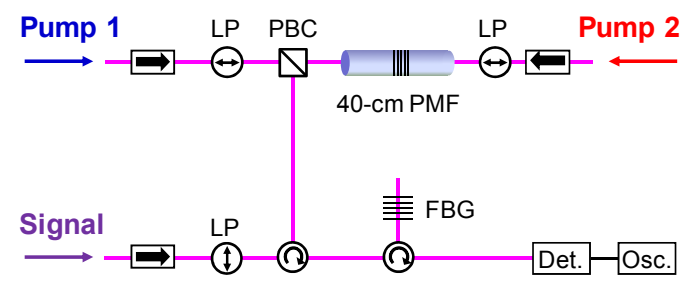

Fig. 3: Experimental layout to realize the delaying of a continuous data stream. LP; linear polarizer, PBC; polarization beam coupler, FBG; fiber Bragg grating.

the pump pulses, the dynamic Brillouin gratings are periodically created along the fiber with a periodicity of $4 \mathrm{~ns}$, since the pulses contrapropagate. We therefore placed a $60 \mathrm{~cm}$ section of the PM fiber in a heating chamber at a temperature of $62{ }^{\circ} \mathrm{C}$, leading to a small increment of the Brillouin frequency shift by $40 \mathrm{MHz}$ that makes thus possible the interaction along this only segment. However, as a consequence of the intrinsic thermal dependence of the fiber birefringence, the overall shift of the dynamic grating reflection spectrum turns out to be larger and is observed to be $\sim 2 \mathrm{GHz}$. As a result, by a proper spectral positioning of the signal, only the grating generated in the heating area can be selectively addressed.

Light from a distinct DFB laser is fast modulated through an external electro-optic modulator, generating a signal pulse train of $840 \mathrm{ps}$ duration at a repetition rate of $4.4 \mathrm{~ns}$. The probe frequency is precisely controlled so as to be spectrally well centered on the resonance of the grating generated in the heating section. Then the signal pulse is orthogonally polarized with respect to the two pumps and is delivered into the fiber via a polarization beam fiber coupler. Unlike our previous experiment, a temporal synchronization between pump and signal pulses is not necessarily required since the dynamic grating is held constant at a fixed position in the fiber

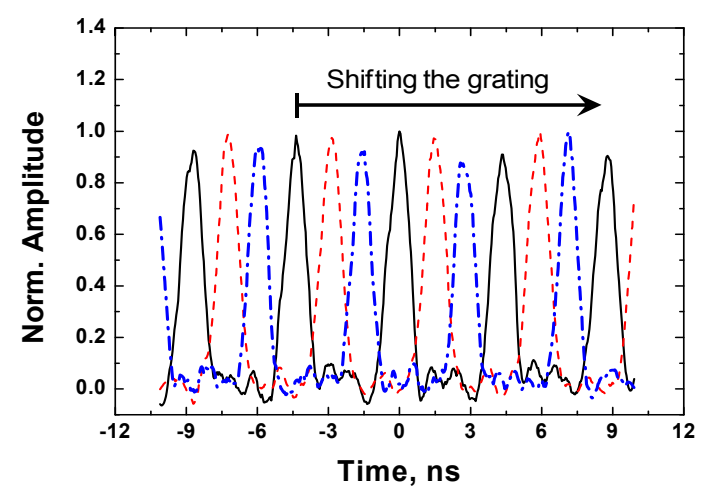

Fig. 4: Time waveforms of the back-reflected signal pulse train for different grating positions. 


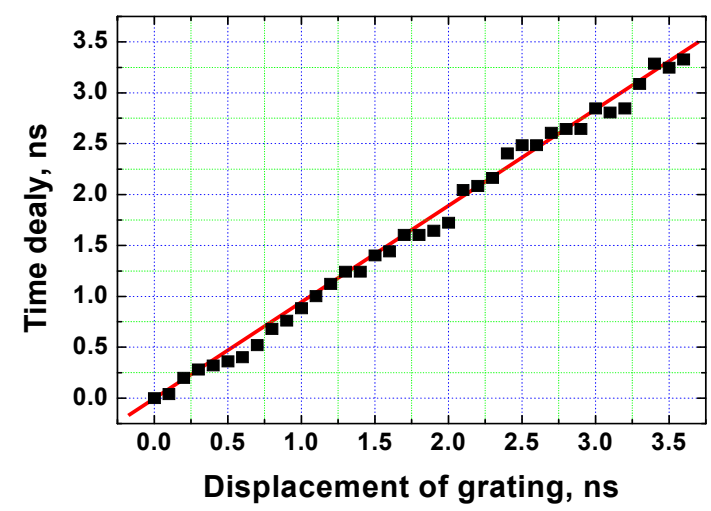

Fig. 5: The achieved pulse delays as a function of displacement of grating position in the fiber.

and continuously reflects the signal.

The normalized time waveforms of the reflected signal pulse train were measured and recorded on a fast oscilloscope while shifting the grating position, as shown in Fig. 4. The signal pulses were clearly delayed without significant signal distortion while the grating was shifted along the fiber by $1.5 \mathrm{~ns}$ steps. To evaluate the amount of signal delay the peak point of the pulse was determined. Fig. 5 shows the achieved time delay as a function of grating position, clearly showing that the signal delay has a linear dependence on the grating position with a slope efficiency of 0.95 . The largest time delay induced by the proposed scheme was $3.3 \mathrm{~ns}$, corresponding to a fractional delay of 4 . This ratio is basically limited up to 4.7 for 840 ps since the maximum achievable time delay is physically determined by the fiber length.

We also tested the feasibility of microwave signal delaying through this type of delay line. For this experiment, instead of pulse modulation, the signal wave was sinusoidally modulated at $500 \mathrm{MHz}$ through an external electro-optic modulator without modifying the set-up. An adequate $\mathrm{DC}$ bias was applied to the modulator in order to completely suppress the carrier. Consequently, only two first-order sidebands were present at the modulator output, generating a microwave signal at $1 \mathrm{GHz}$. The signal frequency was also precisely tuned for a perfect centering on the grating resonance to obtain maximum reflections of this two-tone wave. The back-reflected microwave signal from the grating was recorded on the same oscilloscope and the peak of the sine wave was used to determine the generated phase shifts, as shown in Fig. 6 . The largest achieved phase shift was 620 degree (equivalent to $3.4 \pi$ phase shift) for an equivalent spatial displacement of $2.3 \mathrm{~ns}$. The observed nonlinear response of the phase shift is possibly caused by a non-

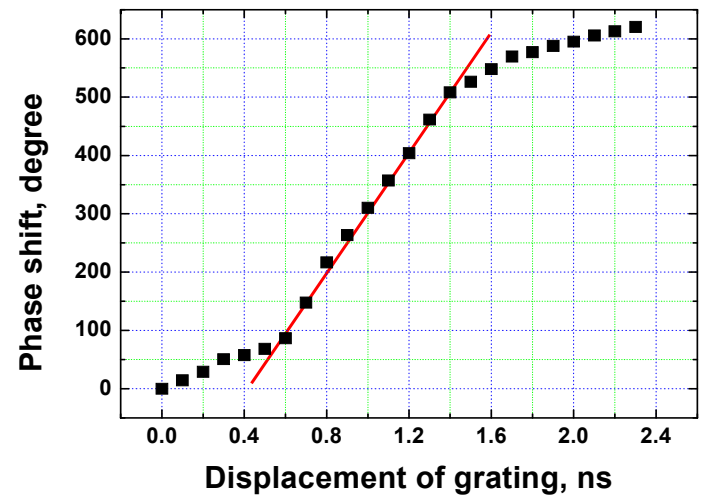

Fig. 6: Measured phase shifts of $1 \mathrm{GHz}$ microwave signal as a function of grating position.

uniformity of the fiber birefringence since the variation of birefringence spectrally shifts the grating resonance and hence the phase shift induced by the spectral resonance. As a result, the differential phase shift experienced by the two optical tones is not identical, thus degrading the linearity of this system and reducing the maximum achievable phase shift.

\section{Conclusions}

We have experimentally demonstrated that reflectors based on dynamic Brillouin grating in polarization maintaining fibers can be implemented to efficiently delay data streams and microwave signals, realizing long delays with low distortion in short fibers, thus with ideal reconfiguration time.

The authors would like to acknowledge the support from the European Community's Seventh Framework Programme [FP7/20072013] under grant agreement $n^{\circ} 219299$ (GOSPEL project) and the Swiss National Science Foundation through project 200020121860.

\section{References}

1 J. B. Khurgin and R. S. Tuchker, Slow Light: Science and applications (CRC Press, Boca Raton, 2009).

2 R. S. Tucker et al., J. Lightwave Technol. 23, 4046 (2005).

3 R. W. Boyd et al., Phys. Rev. 71, 023801 (2005).

4 J. E. Sharping et al., Opt. Express 20, 7872 (2005).

5 M. Fok et al., J. Lightwave Technol. 26, 499 (2008).

6 L. Thévenaz et al., Comptes Rendus Physique 10, 1008 (2009).

7 N. Primerov et al., Proc. OFC'2010, OWF6, (2010).

$8 \mathrm{M}$. Nikles et al., J. Lightwave Technol. 15, 1842 (1997). 